Shoshan, Maria

[27]

\section{Cisplatin induces the pro-apoptotic conformation of bak in a delta-MEKK1- dependent manner}

Aleksandra Mandic, Kristina Viktorsson, Johan Hansson, Stig Linder \& Maria Shoshan

Radiumhemmet Research Laboratory, CCK, Karolinska Institute, S-171 76 Stockholm, Sweden mechanisms or processes.

Shoshan, M.C. [28]

\section{BAK, BAX and p53 proteins in the apoptotic response to cisplatin}

Cisplatin induces the pro-apoptotic conformation of the Bcl-2 family protein Bak in four out of four human melanoma cell lines, whereas an effect on the pro-apoptotic conformation of Bax was seen in only one (FM55 cells). Expression of a kinase-inactive fragment of MEKK1 (dnMEKK) efficiently blocked the modulation of Bak and cytochrome $c$ release, whereas DEVDase activation and nuclear fragmentation were reduced by half. Expression of a kinase-active MEKK1 fragment (dpMEKK) was sufficient to modulate Bak in three out of four cell lines, whereas an effect on Bax was seen only in FM55 cells. In the DFW cell line, dpMEKK stimulated a similar degree of Bak modulation as cisplatin but did not induce nuclear fragmentation. Together with the knowledge that dnMEKK was insufficient to block apoptosis completely, this finding indicates that cisplatin may induce apoptosis by means of additional mechanisms. We conclude that a proapoptotic MEKK1 pathway is necessary for cisplatin-induced Bak modulation, and that this modulation represents one of several cisplatin-induced apoptotic

Mandic, A., Viktorsson, K., Varsanyi, M., Hansson, J., Linder, S. \& Shoshan, M.C.

Radiumhemmet's Research Laboratory, Cancer Center Karolinska, Karolinska Institute, S-171 76 Stockholm, Sweden

Genotoxic damage, as induced by cisplatin, has been reported to lead to upregulation of pro-apoptotic members of the Bcl-2 family. We have here examined the pro-apoptotic Bcl-2 proteins Bak and Bax in human melanoma cells treated with cisplatin. Expression of Bak was not increased by this treatment; instead, equitoxic doses of cisplatin were found to induce the pro-apoptotic conformation of Bak in all human melanoma cell lines tested, irrespective of p53 status. Unlike Bak modulation, cisplatin-mediated modulation of Bax was seen in only one of the p53 wildtype cell lines. The upstream regulation of Bak is not known. We show that expression of a kinase-inactive fragment of the stress-activated kinase MEKK1 (dnMEKK) blocks modulation of Bak and apoptosis. Activation of the downstream kinases JNK1-2, which regulate activity of transcription factor c-Jun, has been shown to be involved in cisplatin-induced apoptosis. Although dnMEKK inhibited apoptosis, JNK1-2 activation was not blocked. Conversely, expression of a kinase-active MEKK1 fragment (dPMEKK) was able to modulate Bak and to induce apoptosis in a cell-line-dependent manner. We conclude that a pro-apoptotic MEKK1 pathway is necessary for cisplatin-induced Bak modulation, and that this modulation represents one of several cisplatin-induced apoptotic mechanisms/processes.
Sinibaldi, Dominic

[29]

\section{Defining a molecular fingerprint of STAT3- regulated genes associated with oncogenesis using microarray technology and novel statistical methods}

Dominic Sinibaldi, Roy Garcia, Greg Bloom, Shrikant Mane, Peter Geiser, Susan Minton, Carlos Muro-Cacho, Emmanuel Lazaridis \& Richard J ove

H. Lee Moffitt Cancer Center and Research Institute, and University of South Florida College of Medicine, Tampa Florida 33612, USA

Signal transducers and activators of transcription (STATs) are latent cytoplasmic transcription factors that are involved in normal cytokine and growth factor signaling. Recent studies have shown that certain STAT family members, most notably STAT3, are constitutively activated by various oncoproteins, such as Src. Furthermore, STAT3 signaling is constitutively activated with high frequency in diverse human tumors, including breast carcinoma, head and neck squamous cell carcinoma, multiple myeloma and various leukemias. These findings indicate that downstream target genes of STAT3 contribute to malignant progression. To date a small number of genes have been shown to be regulated by STAT3, including cylcin $D 1, p 21 W A F 1, B c l-x, M c l-1$ and $c-M y c$, which are important in cell cycle control and apoptosis. However, it is likely that additional STAT3-regulated genes participate in oncogenesis. Using Affymetrix microarray chip technology, we have defined a list of genes that are associated with STAT3 activation in cells transformed by the Src oncoprotein and in model human breast carcinoma cell lines. We identified altered gene expression attributed to STAT3 by examining multiple cell lines with different levels of STAT3 activity. The list was refined by altering the STAT3 activation status of various cell lines following several approaches, including the use of pharmacologic inhibitors of STAT3 signaling. We employed a range of statistical approaches to identify genes that most accurately correspond to STAT3 activity in the context of oncogenesis. We have defined a molecular fingerprint of candidate STAT3-regulated genes that potentially contribute to malignant progression of breast cancer.

Sinnett, Daniel

[30]

\section{A detailed transcript map of the human chromosome 12p12.3 tumor suppressor locus: the usefulness of an integrative approach}

\section{Alexandre Montpetit, Nathalie Trudel \& Daniel Sinnett}

Charles-Bruneau Cancer Centre, Division of Hemato-Oncology, Research Center, Ste. Justine Hospital, Montreal, Quebec, Canada

Allellic loss of chromosome 12p is a frequent event in childhood acute lymphoblastic leukemia. This region is also deleted in several other hematological malignancies and in a variety of solid tumors, suggesting the presence of a tumor suppressor gene. The chromosomal region containing this suppressor locus was narrowed down to an interval of approximately 750 kilobases delimited by D12S98 and D12S358. Since no known candidate gene was found, we initiated the construction of a detailed transcription map, focusing on a contig of four overlapping BACs. We applied a strategy integrating several complementary approaches: (1) application of computerbased data-mining tools to the existing genomic sequence $(750 \mathrm{~kb})$ derived form the BAC contig; (2) deployment of exon amplification and expressed sequence tag resources to identify putative complementary DNAs; (3) determination of the general expression pattern by polymerase chain reaction with reverse transcription and 